INPLASY

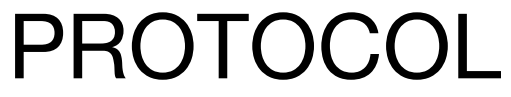

To cite: Jin et al. The prognostic value of EMT phenotype of circulating tumor cells in breast cancer patients: a meta-analysis. Inplasy protocol 202040123. doi: 10.37766/inplasy2020.4.0123

Received: 20 April 2020

Published: 20 April 2020

Corresponding author: Jin Yanling

13893402351@163.com

Author Affiliation:

Lanzhou University

Support: Izujbky-2019-it12 (YL.J)

Review Stage at time of this submission: Preliminary searches.

Conflicts of interest: We declare no conflicts of interest.

\section{The prognostic value of EMT phenotype of circulating tumor cells in breast cancer patients: a meta-analysis}

\author{
Jin, Y1; Ge, L2; Zhao, Y3; Guo, C4; Wei, J5.
}

Review question / Objective: This study aims to investigate the correlation between EMT phenotype of CTCs and the prognosis of patients with breast cancer.

Condition being studied: Tumor metastasis is the main cause of cancer related deaths, CTCs play a vital role in breast cancer metastasis and recurrence. Previous studies indicated that CTCs regarded as a prognosis value in breast cancer, however, CTCs have heterogeneity due to EMT process, including three CTCs phenotype: epithelium, hybrid type and mesenchymal phenotype. The prognostic significance of CTCs with EMT phenotype in breast cancer patient is controversial, and we will identify and synthesise trials to investigate whether EMT phenotype of CTCs isolated in peripheral blood plays a prognosis value in breast cancer patients, we will also examine the risk of bias and heterogeneity between studies.

INPLASY registration number: This protocol was registered with the International Platform of Registered Systematic Review and Meta-Analysis Protocols (INPLASY) on 20 April 2020 and was last updated on 20 April 2020 (registration number INPLASY202040123).

\section{INTRODUCTION}

Review question / Objective: This study aims to investigate the correlation between EMT phenotype of CTCs and the prognosis of patients with breast cancer.

Condition being studied: Tumor metastasis is the main cause of cancer related deaths,
CTCs play a vital role in breast cancer metastasis and recurrence. Previous studies indicated that CTCs regarded as a prognosis value in breast cancer, however, CTCs have heterogeneity due to EMT process, including three CTCs phenotype: epithelium, hybrid type and mesenchymal phenotype. The prognostic significance of CTCs with EMT phenotype in breast cancer 
patient is controversial, and we will identify and synthesise trials to investigate whether EMT phenotype of CTCs isolated in peripheral blood plays a prognosis value in breast cancer patients, we will also examine the risk of bias and heterogeneity between studies.

\section{METHODS}

Participant or population: Individuals of any age with breast cancer.

Intervention: Breast cancer patients were detected CTCs with mesenchymal markers positive in peripheral blood.

Comparator: Breast cancer patients were detected CTCs with mesenchymal markers negative in peripheral blood.

Study designs to be included: Prospective studies or retrospective studies and secondary analysis of randomised controlled trials (RCTs).

Eligibility criteria: 1) EMT markers (epithelial markers, mesenchymal markers) of CTCs were examined in patients with breast cancer; 2) Hazard ratio of survival between patients with mesenchymal markers positive CTCs and mesenchymal markers negative CTCs was reported, or provided a completely Kaplan-Meier curve.

Information sources: The following electronic databases will be retrieved from database inception to April 2020: Pubmed, EMBASE, Cochrane and China National Knowledge Infrastructure (CNKI). The reference lists of relevant articles (including review articles) that met the inclusion criteria will be screened.

Main outcome(s): Overall survival (OS) or recurrence-free survival (RFS), and/or poor progression-free survival (PFS).

Data management: Data will be extracted from studies that are suitable for inclusion criteria, including name of the first author, year of publication, study design, Number of participants, demographic data of subjects, tumor stage, time of blood sampling, EMT markers detection methods, number of patients with mesenchymal markers positive CTCs, number of patients with mesenchymal markers negative CTCs, OS, PFS, and/or DFS, clinicopathological characteristics (age, sex, risk factors, and metastasis). Data extraction was performed by 2 independent reviewers, and a third reviewer was consulted for any uncertainties.

Quality assessment / Risk of bias analysis: The Newcastle Ottawa Quality Assessment Scale (NOS) will be used to assess the quality of cohort studies, and the selected full-text articles will be rated as good, fair or poor. Two reviewers will individually appraise the quality of included studies, a third reviewer will resolve the disagreements through discussion.

Strategy of data synthesis: RevMan V.5.3soft ware will be used to carry out statistical analysis. Outcome values estimate as descriptive statistics and $95 \%$ confidence intervals. Heterogeneity across eligible studies will be checked by $I^{2}$ statistic, a fixed-effects model will be chosen when $\mathrm{I}^{2} \leq 50 \%$, On the other hand, a random-effects model will be chosen when $\left.\right|^{2}>50 \%$.

Subgroup analysis: The origin of substantial heterogeneity is explored by subgroup analyses, according to the sample size, cut-off value, histological type, therapy methods, tumor metastasis, and overall risk of bias.

Sensibility analysis: The leave-one-out approach will be used to perform sensitivity analysis. We will also perform a fixed effect model meta-analysis to observe the robustness of results.

\section{Country(ies) involved: China.}

Keywords: Breast neoplasms; Circulating Tumor Cell; Epithelial-Mesenchymal Transition; prognostic factor; metaanalysis.

Contributions of each author: 
Author 1 - Yanling Jin designed the research, managed the data and drafted the manuscript.

Author 2 - Long Ge provided statistical expertise.

Author 3 - Yingying Zhao developed search strategy, retrieved literature and extracted data.

Author 4 - Chen Guo developed search strategy, retrieved literature and extracted data.

Author 5 - Jing Wei contributed to the development of the risk of bias assessment strategy, the subgroup analysis and sensibility analysis. 